\title{
Calidad de las estadísticas de mortalidad en Chile, 1997-2003
}

\author{
M Loreto N úñez F1,3a, M G loria Icaza $\mathrm{N}^{2,3 b}$. \\ Q uality of M ortality statistics in Chile, \\ 1997-2003
}

Background: Death certification is the basis for the study of mortality patterns and the identification of leading causes of death. These data are used for planning health policies and prevention programs. Aim: To evaluate the quality of mortality statistics in Chile from 1997 and 2003. Material and methods: Mortality data from 1997 to 2003 available at the Chilean Ministry of Health was used. Quality of information was evaluated using the percentage of certifications done by physicians and ill-defined causes of death according to rural or urban residence, medical services, age and gender. Results: Death certification done by physicians increased from $97.6 \%$ to $99.0 \%$ in the studied period. The greatest increase was observed in rural areas, from $89.9 \%$ to $94,8 \%$. There was a steady reduction in the percentage of ill-defined causes of deaths, from $4.7 \%$ in 1997 to $2.8 \%$ in 2003 . The percentage of ill-defined causes had a great variation among the different medical services. The variation went from $1.5 \%$ in Magallanes to $13.7 \%$ in Araucania Sur. There was an inverse linear correlation between death certification by physicians and ill-defined conditions. Conclusions: Quality of Chilean mortality statistics has improved over time. Nevertheless, there is still space for enhancement for the appropriate use of this information (Rev Méd Chile 2006; 134: 1191-6).

(Key w ords: Cause of death; Data collection; Mortality; Registries; Vital statistics)
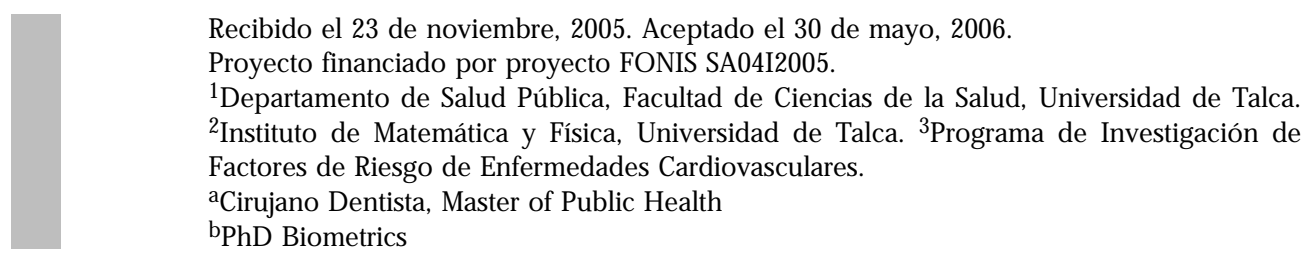

L a necesidad de registrar y analizar el comportamiento de las defunciones según sus causas, ha existido desde hace siglos. El conocimiento de la mortalidad en cuanto a su frecuencia, distribución por región, edad, sexo y algunas características socioeconómicas asociadas, así como sus cambios en el tiempo, es usado principalmente para: investigar los factores causales de enfermedades y muertes, evaluar la situación de salud de la población en diversos períodos, evaluar los resultados de los programas de salud aplicados, planear los servicios de salud que requiere la

población y aplicar medidas para prevenir las enfermedades y las muertes prematuras ${ }^{1}$. Para hacer uso eficiente de esta información es necesario monitorear periódicamente la calidad de las estadísticas de mortalidad ${ }^{2}$. Tradicionalmente, se ha medido la calidad de las estadísticas de mortalidad a partir de la cobertura de certificación médica y de la calidad de dicha certificación ${ }^{3,4}$.

El sistema de estadísticas vitales en Chile es centralizado y lo integran el Instituto Nacional de Estadísticas, el Servicio de Registro Civil e Identificación y el Ministerio de Salud. En Chile es obligatorio emitir un certificado de defunción para proceder a la sepultación, lo que permite contar con certificado de defunción para la gran mayoría 
de los decesos. Dentro del contexto latinoamericano, Chile ha sido considerado como un país con relativamente buenas estadísticas vitales, ya que posee tradición en la recolección de datos ${ }^{3}$.

El objetivo de este trabajo es evaluar la calidad de la información de mortalidad proveniente de las estadísticas vitales en Chile durante el período 1997-2003, últimos años disponibles con la décima revisión de la Clasificación Estadística Internacional de Enfermedades (CIE-10) ${ }^{5}$.

Este trabajo se enmarca dentro del proyecto FONIS «Atlas de mortalidad por enfermedades cardiovasculares, Chile 1997-2003».

\section{MATERIAL Y MÉTODO}

La información utilizada en este estudio se obtuvo de las bases de datos de defunciones del período 1997-2003, clasificadas de acuerdo a: sexo, edad, año de defunción, servicio de salud, causa básica de defunción, área urbana-rural y tipo de certificación.

Para evaluar la calidad de la información de mortalidad, se consideraron las variables de certificación médica y causas mal definidas. Las causas mal definidas analizadas fueron los síntomas, Signos y Hallazgos Anormales Clínicos y de Laboratorio, no Clasificados en Otra Parte» del Capítulo XVIII, códigos R00-R99, de la CIE-10. Estas variables fueron analizadas por área urbanorural, sexo, servicio de salud y por edad.

Se realizó análisis estadístico de los datos que incluye: tablas de frecuencia y contingencia, gráfi- cos ad-hoc y análisis de correlación. Para el manejo y análisis de datos se utilizó el programa SAS versión 9.1.3.

\section{RESULTADOS}

En el peńodo en estudio, la cobertura de la certificación médica de defunciones en Chile se incrementó de 97,6\%, en 1997, a 99,0\% en 2003 (Figura 1). Sin embargo, la cobertura difiere entre las áreas de residencia urbana y rural. En el año 1997, en las áreas urbanas, 99,2\% de los fallecimientos fueron certificados por médico, en cambio, en el sector rural sólo lo fue en $89,9 \%$. A través del tiempo, ambas cifras han mostrado avances positivos. En 2003, la certificación médica urbana alcanzó 99,6\% y la certificación médica rural 94,8\% (Figura 1). Llama la atención que la certificación médica rural presentó un aumento notable en 2002, para luego retomar su tendencia histónica en 2003. En los años estudiados, la certificación médica fue de $98,5 \%$, siendo similar en hombres $(98,6 \%)$ y mujeres $(98,4 \%)$.

$\mathrm{Si}$ se consideran las causas mal definidas contenidas en el certificado de defunción, se observa que en 1997 hubo 4,7\% de causas mal definidas. Esta cifra desciende a 2,8\% en 2003 (Figura 2). Tal como sucede con la cobertura de certificación médica, el porcentaje de causas mal definidas difiere entre área de residencia urbana y rural. En 1997, se encontró $3,1 \%$ de causas mal definidas en el área urbana y $13,2 \%$ en el sector rural. La evolución temporal de esta variable ha sido positiva. En 2003, el porcentaje
Figura 1. Porcentaje de certificación médica total, rural y urbana de defunciones, Chile 1997-2003.

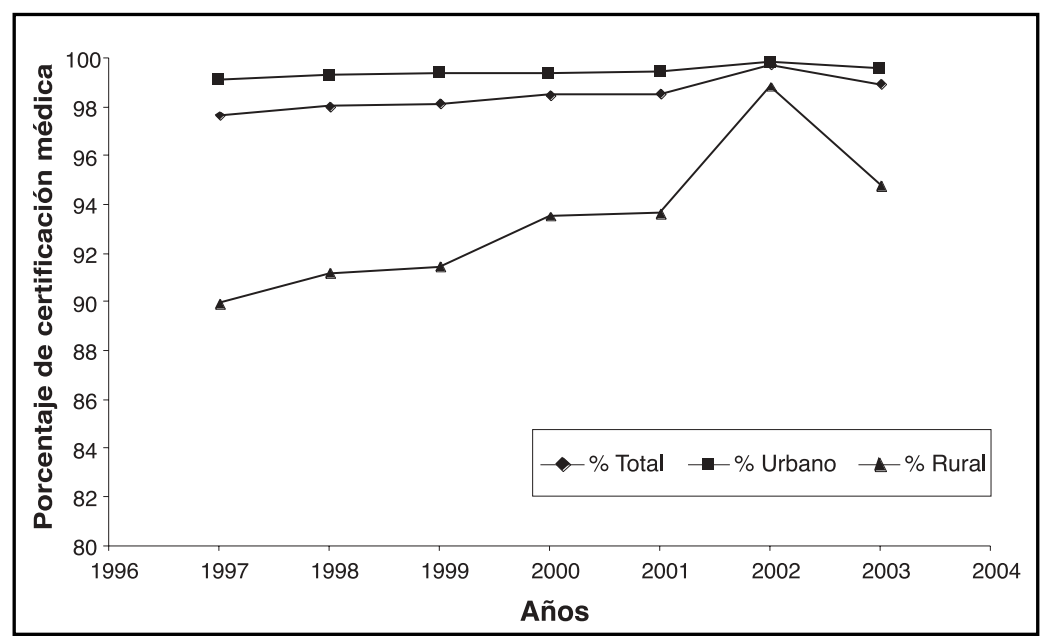


de causas mal definidas en el área urbana fue de 2,1\% y en el área rural fue de 7,5\%, en concordancia con la tendencia observada para la certificación médica (Figura 2). El porcentaje de causas mal definidas para el período fue de 3,8\%, siendo 3,3\% en hombres y $4,5 \%$ en mujeres. La brecha entre sexos ha disminuido durante el período en estudio.

El descenso en el número de causas mal definidas que se observa a partir del año 2000 (Figura 2), se puede atribuir a que ese año se especificaron normas de codificación y de procesamiento que se aplican desde entonces en el marco de la reorganización del Departamento de Estadísticas e Información en Salud (DEIS) del Ministerio de Salud. Así, por ejemplo, todos los registros en que la causa básica seleccionada es del capítulo XVIII, son objeto de reparo con consulta al médico que certificó y, según el caso, recurriendo a distintas fuentes de información como la historia clínica, egresos hospitalarios, autopsias médico legales, la vigilancia de accidentes del trabajo o la notificación de enfermedades de declaración obligatoria.

El porcentaje de causas mal definidas por servicio de salud presenta un amplio rango de variación (Figura 3), con un máximo de 13,7\% en el Servicio de Salud Araucanía Sur y un mínimo de $1,5 \%$ en el Servicio de Salud Magallanes. El porcentaje nacional de causas mal definidas, que fue de 3,8\% para el peńodo, fue sobrepasado por nueve servicios de salud.

Al investigar la relación entre el porcentaje de causas mal definidas y el porcentaje de certificación médica, se observó que existe una relación lineal

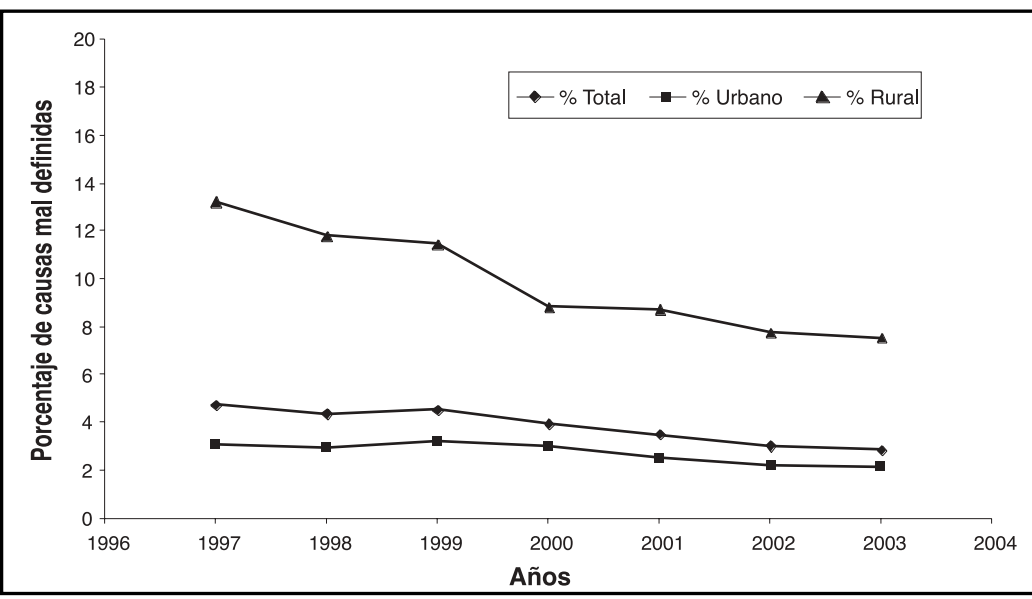

Figura 2. Porcentaje de causas mal definidas total, rural y urbana de defunciones, Chile 19972003.

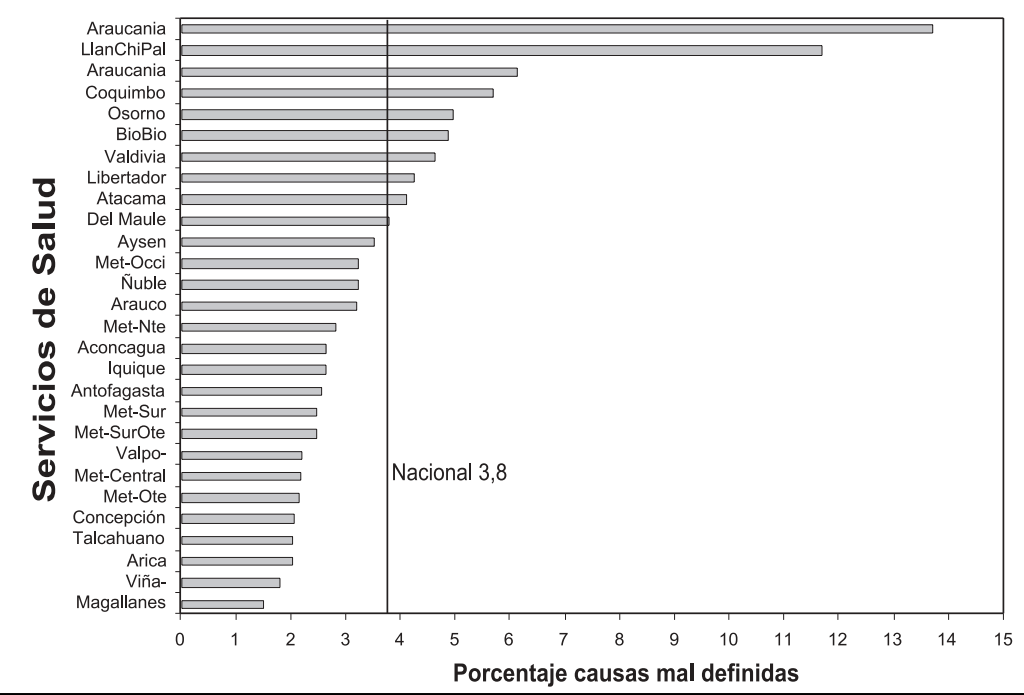

Figura 3. Porcentaje de defunciones por causas mal definidas, según Servicio de Salud, Chile 1997-2003. 
Figura 4. Relación entre el porcentaje de certificación médica total y porcentaje de causas mal definidas en defunciones, Chile 1997-2003.
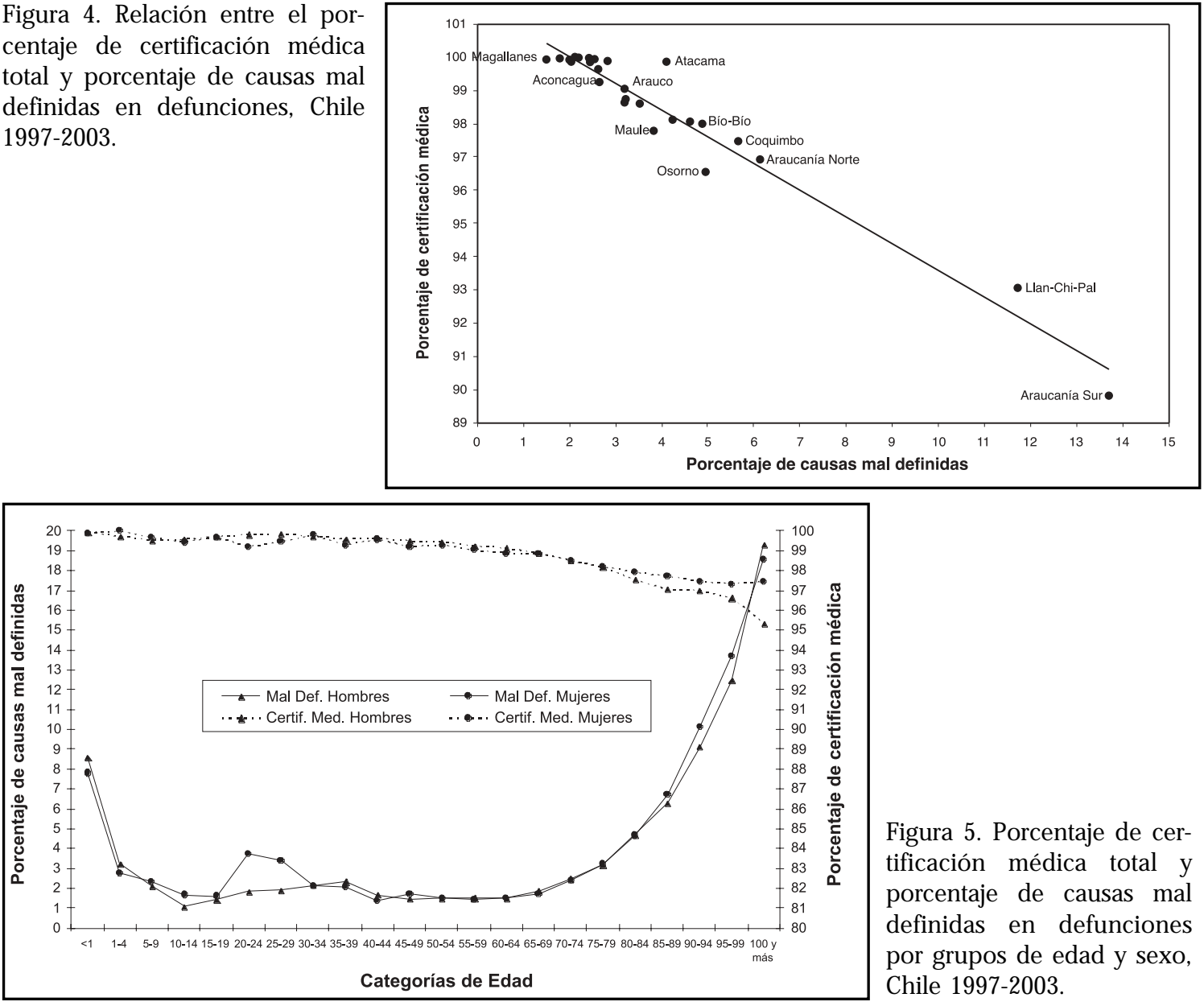

Figura 5. Porcentaje de certificación médica total y porcentaje de causas mal definidas en defunciones por grupos de edad y sexo, Chile 1997-2003.

inversa ( $r=0,977$, valor $p \varangle 0,001$ ) (Figura 4). Los Servicios de Salud Araucanía Sury Llanquihue-ChiloéPalena, son los que tienen la más baja cobertura de certificación médica y el más alto porcentaje de causas mal definidas. Para el peńodo en estudio, estos aparecen con valores extremos (mayores a 1,5 veces el rango entre cuartiles) con respecto al resto de los servicios. Sin embargo, todos los servicios de salud muestran una tendencia a aumentar el porcentaje de certificación médica y a disminuir las causas mal definidas durante el peńodo. Los servicios con mejores resultados, en el quintil más alto, en cuanto a ambos indicadores de calidad son, en orden decreciente, los servicios de salud de: Magallanes, Viña del Mar-Quillota, Talcahuano, Arica, Metropolitano Oriente y Metropolitano Central.

El análisis por grupos de edad (Figura 5), muestra que el porcentaje de certificación médica tiene su nivel más alto en los menores de un año $(99,9 \%)$ y tiende a disminuir con la edad. Esta disminución es más acentuada en los hombres que en las mujeres, comenzando a diferenciarse desde los 75 años en adelante, llegando a ser de $97,0 \%$ en el grupo de 100 años y más. Se observa una leve diferenciación por sexos en este grupo siendo el porcentaje de certificación médica en hombres de $95,4 \%$ y en mujeres de $97,5 \%$.

A excepción del grupo de menores de un año, el porcentaje de causas mal definidas tiene un comportamiento inverso a la certificación médica. El porcentaje más alto de causas mal definidas se encuentra en el grupo de 100 años y más con 18,7\%, no observándose una gran diferencia entre hombres $(19,3 \%)$ y mujeres (18,6\%). Es interesante destacar que en los menores de un año, el porcentaje de causas mal definidas es alto, a pesar de ser alto el porcentaje de 
certificación médica. Esto se debe precisamente a que, de las causas mal definidas de los menores de un año, 69,2\% comesponde a R95 (síndrome de la muerte súbita infantil) que es aceptado como entidad causante de muertes en este grupo de edad por los médicos, existiendo abundante literatura e hipótesis de causalidad al respecto. Si restamos a las causas mal definidas en menores de un año, las defunciones por muerte súbita, el porcentaje de mal definidas en menores de un año seńa de 2,5\%, porcentaje menor que el promedio nacional para el período $(3,8 \%)$ y cercano a los grupos etarios de 1 a 9 años.

Otro hecho relevante es que en los mayores de 65 años el código R54 (senilidad) representa un tercio $(34,4 \%)$ de las causas mal definidas; de estos diagnósticos, 99,7\% fue realizado por médicos, lo que indica que esta causa mal definida no puede ser atribuida a la falta de certificación médica de la defunción.

Del total de defunciones sin certificación médica en el período de estudio, 96,5\% son por causas mal definidas, es decir una mayoría. Sin embargo, de las causas mal definidas en el período, 62,5\% fueron certificadas por médicos. Es decir, persiste entre las causas mal definidas un gran porcentaje que sí fue certificado por médicos.

\section{DisCUSIÓN}

La tendencia ascendente de la cobertura de certificación médica en Chile, observada en este estudio, ya había sido reportada para el peńodo 1970-1990, donde la certificación médica de defunciones aumentó de $81,0 \%$ a $95,0 \%{ }^{6}$. En un estudio publicado por Haynes, que comprende datos de 1976 a 1978, se observó que 87,0\% de las defunciones en Chile fueron certificadas por médico ${ }^{7}$. El estudio de Haynes muestra que el porcentaje de certificación médica variaba significativamente entre las provincias de Chile; en las predominantemente rurales, la certificación médica osciló entre 50,0\% y 75,0\%, a diferencia de las provincias urbanas, donde la certificación médica bordeaba 95,0\%. Publicaciones de otros países de América Latina muestran que Chile tiene una situación similar a Panamá en el año 1995, en que 89,4\% de las defunciones tuvieron certificación médi$\mathrm{ca}^{8}$. Por otro lado, estas cifras se diferencian positivamente de la situación de Paraguay, que en el peníodo 1996-1999 entre 51,5\% y 54,0\% de las muertes registradas contaron con certificación médica ${ }^{9}$.
Al evaluar el porcentaje de certificación médica por grupo de edad, se observan diferencias con el estudio realizado por Castillo, con datos de 1983, donde se observó que los grupos de edades extremas (menos de 14 y más de 65 años) son los que presentan los porcentajes de certificación médica más bajos (85,5\% y $82,8 \%$, respectivamente) y la mayor certificación médica se da en el grupo de 15-24 años, donde $94,0 \%$ de las defunciones fueron certificadas por médicos ${ }^{10}$. Se constata que en el período 19972003 cambió favorablemente la situación en los menores de 14 años, donde la certificación médica es máxima para luego descender progresivamente según aumenta la edad, tendencia que se acentúa en el sexo masculino a partir de los 75 años.

Según el estudio de Castillo, en 1983 Chile tenía 9,0\% de defunciones debidas a causas mal definidas, pero con marcadas diferencias por servicio de salud, con un máximo de 31,8\% en el Servicio de Llanquihue-Chiloé-Palena y un mínimo de 0,8\% en Magallanes ${ }^{11}$. En 1990, el porcentaje de causas mal definidas descendió a $6,0 \% 6$, en consecuencia, el 2,8\% observado en este estudio para el año 2003, revela importantes avances a lo largo del tiempo. Llama la atención que los servicios histónicamente con deficiencias en sus registros son Llanquihue-ChiloéPalena y Araucanía, que en el año 1983 no estaba dividido en Sur y Norte. Actualmente, el Servicio Araucanía Sur es el que presenta el porcentaje más alto de causas mal definidas.

Según reportes de la Organización Panamericana de la Salud, se observan diferencias importantes en los países de las Américas. Alrededor del año 2000, las defunciones por causas mal definidas alcanzaron 1,3\% en Canadá, 6,6\% en Argentina y $14,8 \%$ en Brasil ${ }^{12}$. Si se consideran estas cifras, Chile se encontraría relativamente cercano a la situación de Canadá. Tradicionalmente, Chile ha sido considerado como un país con relativamente buenas estadísticas comparado con otros países latinoamericanos ${ }^{3,4}$. Sin embargo, Mathers, en el año 2005, hace un análisis global de causas mal definidas en el cual clasifica a Chile dentro del grupo de países con estadísticas de mortalidad de calidad media. En esta misma categoría se encuentran países de Latinoamérica como: Belice, Brasil, Colombia, Costa Rica, El Salvador, Guatemala, Panamá y Uruguay. En la categoría de calidad superior se encuentran Canadá, Cuba, México, Estados Unidos y Venezuela ${ }^{13}$. 
En Chile se ha hecho esfuerzos por estandarizar el modo correcto de llenar el certificado médico de defunción ${ }^{14,15}$, acción que ha tenido efecto en el mejoramiento de la calidad de los datos como se muestra en este estudio. No obstante estas mejorías, Chile está distante de los países desarrollados tales como Canadá y Estados Unidos de Norteamérica que presentan cifras que bordean $1 \%$ de causas mal definidas ${ }^{16}$; por lo que es necesario continuar realizando esfuerzos masivos tendientes a mejorar la calidad de la información de causas de muerte, a

\section{REFERENCIAS}

1. Organización Panamericana de ia Salud. Clasificación internacional de enfermedades, 2003. Disponible en: ais.paho.org/cie/index.htm?xml=antecedents.htm [Consultado 16 de marzo 2006].

2. Benavides F, Bolumar F, Peris R. Quality of death certificates in Valencia, Spain. Am J of Public Health 1989; 79: 1352-4.

3. Instituto Nacional de Estadísticas. Anuario de Demografía, 2000.

4. Jaspers-Faijer D, Orelina H. Evaluación del uso de las estadísticas vitales para estudios de causas de muerte en América Latina. Notas de Población, Número 60, CELADE, Santiago, Chile, 1994.

5. Organización Mundial de la Salud. Clasificación estadística internacional de enfermedades y problemas relacionados con la salud. - 10aㅡ revisión. v. 3. Lista tabular. Washington, D.C.: OPS. (Publicación científica 554), 1995.

6. Taucher E, Albala C, Icaza G. Adult Mortality from Chronic Diseases in Chile, 1968-90. En: Adult Mortality in Latin America. Editado por Ian M. Timæus, Juan Chackiel y Lado Ruzicka. Clarendon Press, Oxford, 1996; 253-75.

7. HAYNES R. The geographical distribution of mortality by cause in Chile. Soc Sci Med 1983; 17: 355-64.

8. Organización Panamericana de la Salud. Resumen del capítulo de país de Salud en las Américas, 1998. Disponible en: www.paho.org/spanish/sha/ prflpan.htm [Consultado 13 junio 2005]

9. Organización Panamericana de la Salud. Perfiles de País: Paraguay. Boletín Epidemiológico, vol. 25 № 2, junio 2004. Disponible en: www.paho.org/ spanish/dd/ais/be_v25n2-perfil-paraguay.htm [Consultado 13 junio 2005].

10. CASTILO B, MaRdones G. Certificación médica en los servicios de salud de Chile. Rev Méd Chile 1986; 114: 693-700. través de la enseñanza de las normas del registro, de la importancia de un registro completo y fidedigno y evitar el uso de causas mal definidas ${ }^{12}$.

En resumen, Chile es considerado hoy en día un país con estadísticas de mortalidad de mediana calidad. Las diferencias reportadas en los porcentajes de certificación médica y porcentajes de causas mal definidas, por Servicios de Salud, por área urbanorural, por sexo y por edad, afectan los estudios de mortalidad y causas de muerte, y por lo tanto afectan la toma de decisiones en el sistema de salud.

11. Castilo B, Mardones G. Defunciones por causas mal definidas en los servicios de salud en Chile. Rev Méd Chile 1986; 114: 359-66.

12. SiBAI A. Mortality certification and cause-of-death reporting in developing countries. Bulletin of the World Health Organization, 2004: 82(2). Disponible en www.who.int/bulletin/volumes/82/2/ 83.pdf [Consultado 20 de marzo 2006].

13. Mathers C, Ma Fat D, Inoue M, Rao C, López A. Counting the dead and what they died from: an assessment of the global status of cause of death data. Bull World Health Organ 2005; 83, no.3: 171-177c. Disponible en: www.scielosp.org/ scielo.php ?script=sci_arttext\&pid=S0042$96862005000300009 \& \operatorname{lng}=$ en $\& n r m=$ iso [Consultado 8 de octubre 2005].

14. TAUCher E. Certificado médico de defunciones. Instrucciones para su uso. Documento técnico. Departamento de Informática, Ministerio de Salud, 1992.

15. Instituto Nacional de Estadísticas. De qué mueren los chilenos. Enfoques estadísticos, mortalidad. Boletín informativo del Instituto Nacional de Estadísticas, Número 12, Mortalidad, octubre 2001. Disponible en: www.censo2002.cl/ menu_superior/cuantos_somos/download/ enf_mortalidad.pdf [Consultado 12 de noviembre 2004].

16. Kochanek K, Murphy S, Anderson R, Scott C. Deaths: Final Data for 2000. National Vital Statistics Reports, 2004; 53(5).

\section{Agradecimientos}

Las autoras agradecen a la Dra. Danuta Raj por facilitar las bases de datos de mortalidad para los años 1997 a 2003 y por proporcionar valiosa información referente a algunos resultados encontrados. También agradecen a la Dra. Erica Taucher por su valioso aporte en la redacción de este manuscrito. 